\title{
Nuclear data uncertainty analysis for the Po-210 production in MYRRHA
}

\author{
Luca Fiorito ${ }^{1, *}$, Alexey Stankovskiy ${ }^{2}$, Augusto Hernandez-Solis ${ }^{2}$, Gert Van den Eynde ${ }^{2}$, and Gasper Zerovnik ${ }^{3}$ \\ ${ }^{1}$ Nuclear Energy Agency/Data Bank, 46 Quai Alphonse le Gallo, 92100 Boulogne-Billancourt, France \\ 2 SCK•CEN Belgian Nuclear Research Center, Boeretang 200, 2400 Mol, Belgium \\ ${ }^{3}$ European Commission Joint Research Centre, Retieseweg 111, 2440 Geel, Belgium
}

Received: 7 December 2017 / Received in final form: 7 February 2018 / Accepted: 8 June 2018

\begin{abstract}
MYRRHA is a multi-purpose research reactor able to operate in sub-critical and critical modes and currently in the design phase at SCK•CEN. The choice of LBE was driven by its chemical stability, low melting temperature, high boiling point, low chemical reactivity with water and air and a good neutronic performance. As a drawback, the neutron capture in ${ }^{209} \mathrm{Bi}$ results in the production of ${ }^{210} \mathrm{Po}$, a highly radiotoxic alpha emitter with relatively short half-life ( $\approx 138$ days). The ${ }^{210}$ Po production represents a major safety concern that has to be addressed for the reactor licensing. In this work we used the ALEPH-2 burnup code to accurately calculate the ${ }^{210}$ Po production in a MYRRHA operating cycle. The impact of using different nuclear data libraries was evaluated and the reliability of the results was determined by quantifying the uncertainty of the ${ }^{210} \mathrm{Po}$ concentration. The uncertainty quantification was carried out sampling the currently available nuclear data covariance matrices with the SANDY code. Also, estimates of the sensitivity profiles were obtained with a linear regression approach. The activation yield of the ${ }^{209} \mathrm{Bi}$ neutron capture reaction was assessed as the largest nuclear data source of uncertainty, however the lack of covariances for such data represent a capital drawback for the ${ }^{210}$ Po content prediction.
\end{abstract}

\section{Introduction}

${ }^{210} \mathrm{Po}$ is the last-but-one daughter product of the ${ }^{238} \mathrm{U}$ decay chain, which makes it the only polonium isotope existing in nature. However, because of its relatively short half-life $(\approx 138$ days $)$, it is present only in traces. A pure $\alpha$ emitter, ${ }^{210} \mathrm{Po}$ decays to the stable isotope ${ }^{206} \mathrm{~Pb}$ emitting $5.407 \mathrm{MeV} \alpha-$ particles with a specific activity of $166 \mathrm{TBq} / \mathrm{g}$. The technological interest in ${ }^{210} \mathrm{Po}$ is to be associated to its huge radiotoxicity when ingested or inhaled, since this isotope is retained for weeks in the human body. Its lethal dose from intake is estimated to be much smaller than $1 \mu \mathrm{g}$.

${ }^{210} \mathrm{Po}$ is of great concern for the new generation of nuclear reactors (GEN-IV) involving lead-bismuth eutectic (LBE), where polonium is artificially produced. The major production process is reported in Figure 1 and it involves the ${ }^{209} \mathrm{Bi}$ activation to the ${ }^{210} \mathrm{Bi}$ ground state, which in turn $\beta$-decays to ${ }^{210} \mathrm{Po}$ with a half-life of 5.013 days.

The use of LBE as coolant material in fast reactor systems and/or spallation target in subcritical Accelerator Driven System (ADS) such as Multi-Purpose hYbrid Research Reactor for High-tech Applications (MYRRHA) [1] makes the nuclear data evaluation of

\footnotetext{
* e-mail: lucafiorito.11@gmail.com
}

the ${ }^{209} \mathrm{Bi}$ capture cross section and the associated branching ratio (production yield) to ${ }^{210 g} \mathrm{Bi} /{ }^{210 m} \mathrm{Bi}$ a point of interest. Experimental data are very scarce in the literature (EXFOR), especially in the resonance regions. As a consequence, discrepancies are evident in the evaluated nuclear data, which lead to diverging predictions of the ${ }^{210} \mathrm{Po}$ production in nuclear reactor.

The goals of this paper are to analyze the status of the current evaluated nuclear data for ${ }^{209} \mathrm{Bi}$ and to highlight the differences between evaluations. Then, the effect of the different libraries is studied for the ${ }^{210} \mathrm{Po}$ prediction at the end of a MYRRHA irradiation cycle. Also, a rough uncertainty value is assessed for this quantity by propagating the nuclear data covariances using the SANDY code, based on Monte Carlo sampling.

\section{Polonium production in MYRRHA}

An innovative research reactor MYRRHA [1] is being developed at SCK $\bullet \mathrm{CEN}$, Mol, Belgium. Its major feature is its sub-critical operation mode where the highly-enriched MOX fueled core is coupled to a high-power linear proton accelerator. The accelerator delivers a $4 \mathrm{~mA}$ beam of $600 \mathrm{MeV}$ protons to the LBE spallation target that generates neutrons, thus driving the sub-critical core. The pool reactor 
Table 1. Different channels for the polonium production in the spallation target of MYRRHA.

\begin{tabular}{|c|c|c|}
\hline Isotope & Channel & $\%$ in total production rate \\
\hline \multirow[b]{2}{*}{${ }^{208} \mathrm{Po}$} & ${ }^{209} \mathrm{Bi} \stackrel{(\mathrm{p}, 2 \mathrm{n})}{\longrightarrow}{ }^{208} \mathrm{Po}$ & 98.98 \\
\hline & ${ }^{209} \mathrm{Bi} \stackrel{(\alpha, 5 \mathrm{n})}{\longrightarrow}{ }^{208} \mathrm{At} \stackrel{1.63 \mathrm{~h}}{\longrightarrow}{ }^{208} \mathrm{Po}$ & 1.02 \\
\hline \multirow{2}{*}{${ }^{209} \mathrm{Po}$} & ${ }^{209} \mathrm{Bi} \stackrel{(\mathrm{p}, \mathrm{n})}{\longrightarrow}{ }^{209} \mathrm{Po}$ & 97.33 \\
\hline & ${ }^{209} \mathrm{Bi} \stackrel{(\alpha, 4 \mathrm{n})}{\longrightarrow}{ }^{209} \mathrm{At} \stackrel{5.41 \mathrm{~h}}{\longrightarrow}{ }^{209} \mathrm{Po}$ & 2.67 \\
\hline \multirow[b]{2}{*}{${ }^{210} \mathrm{Po}$} & $\begin{array}{l}{ }^{209} \mathrm{Bi} \stackrel{(\mathrm{n}, \gamma)}{\stackrel{(\alpha, 3 \mathrm{n})}{\longrightarrow}} 210 \mathrm{Bi} \stackrel{5.013 \mathrm{~d}}{\longrightarrow}{ }^{209} \mathrm{At} \stackrel{8.1 \mathrm{~h}}{\longrightarrow}{ }^{210} \mathrm{Po} \\
{ }^{209} \mathrm{Po}\end{array}$ & $\begin{array}{l}99.8 \\
0.18\end{array}$ \\
\hline & ${ }^{208} \mathrm{~Pb} \stackrel{(\mathrm{n}, \gamma)}{\longrightarrow}{ }^{209} \mathrm{~Pb} \stackrel{{ }^{209} \mathrm{Bi}\left(\stackrel{(\mathrm{p}, \gamma)}{\longrightarrow}{ }^{210}{ }^{210} \mathrm{Pbo}\right.}{\stackrel{22.3 \mathrm{y}}{\longrightarrow}}{ }^{210} \mathrm{Bi} \stackrel{5.013 \mathrm{~d}}{\longrightarrow}{ }^{210} \mathrm{Po}$ & $\begin{array}{l}0.02 \\
9.8 \times 10^{-4}\end{array}$ \\
\hline
\end{tabular}

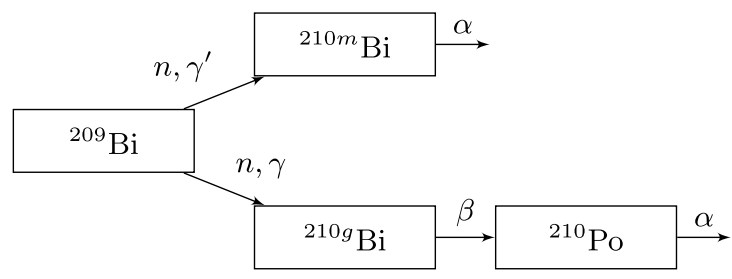

Fig. 1. Scheme of Po production following bismuth activation.

concept implies that the LBE serves as coolant as well. The installation is also assumed to be able to operate in a critical mode as a heavy liquid metal cooled fast reactor, when the beam line is removed and extra MOX fuel assemblies are added to the core periphery. This research facility will serve as a demonstrator for minor actinide transmutation, testing of GEN-IV and fusion reactor materials, producing medical radioisotopes, as well as for fundamental physics research [2].

The use of heavy liquid metal coolants such as lead or LBE is advantageous from the safety and economic point of view. These materials are inert with respect to air and water, have low melting and operating temperatures, as well as low vapor pressure [3]. However, these coolants face several technological challenges. One of the most important issues is the radiological hazard associated with activation of LBE coolant under neutron irradiation. The major concern is the formation of ${ }^{210} \mathrm{Po}$, which has an inhalation and ingestion radiotoxicity among the highest of all the known radionuclides $[4,5]$. In a typical neutron environment characteristic of fast reactors (neutron fluxes at the level of $\left.10^{15} \mathrm{n} /\left(\mathrm{cm}^{2} \mathrm{~s}\right)\right)$ its activity can reach up to $10^{11} \mathrm{~Bq} / \mathrm{kg}$ [6]. In the MYRRHA reactor pool, assuming that all ${ }^{210} \mathrm{Po}$ is retained in $\mathrm{LBE}$, the estimated production of this radioisotope after one irradiation cycle at nominal power would be at the level of $350 \mathrm{~g}$, or $5.5 \times 10^{4} \mathrm{TBq}[7]$. In addition, this polonium isotope contributes the most to the decay heat of LBE, thus determining the strategy of its handling after definitive shutdown of the reactor [8]. During normal reactor operation, most of the polonium (not only ${ }^{210} \mathrm{Po}$, but also ${ }^{208,209} \mathrm{Po}$, which are less radiotoxic due to their longer half-life but equally hazardous since they are $\alpha$-emitters as well) is retained in LBE. This can cause problems during maintenance operations or if coolant leakage occurs. However, some polonium will migrate to the cover gas in the reactor plenum and will diffuse outside the primary system when the reactor is opened for refueling or maintenance. Therefore, a continuous removal of polonium from LBE is foreseen [9].

There are several ways to produce polonium from LBE in MYRRHA. Since the MYRRHA central sub-assembly is occupied by the spallation target, which is not separated from the main LBE pool, the spallation reactions of primary protons and secondary high-energy neutrons with LBE circulating in this assembly also produce polonium isotopes. The contributions of competing reaction channels in the spallation target are summarized in Table 1. The results show that the neutron activation of ${ }^{209} \mathrm{Bi}$ is the main mechanism leading to the ${ }^{210} \mathrm{Po}$ production even in the spallation target, needless to say about the whole core. The other production channels can be neglected either because of the negligible associated cross section, the small particle flux or the unlikely sequence of reactions. Therefore, efforts should concentrate on assessing the uncertainty of the neutron capture reaction rate leading to the formation of ${ }^{210} \mathrm{Bi}$ in the ground state.

\section{Current status of the evaluated ${ }^{209} \mathrm{Bi}(\eta, \gamma)$ cross sections}

The ${ }^{209} \mathrm{Bi}(\eta, \gamma)$ cross section data were taken from the nuclear data libraries JEFF-3.2 [10], ENDF/B-VII.1 [11], JENDL-4.0 [12], TENDL-2015 [13] and BROND$3.1[14]$ and they were compared to estimate analogies 


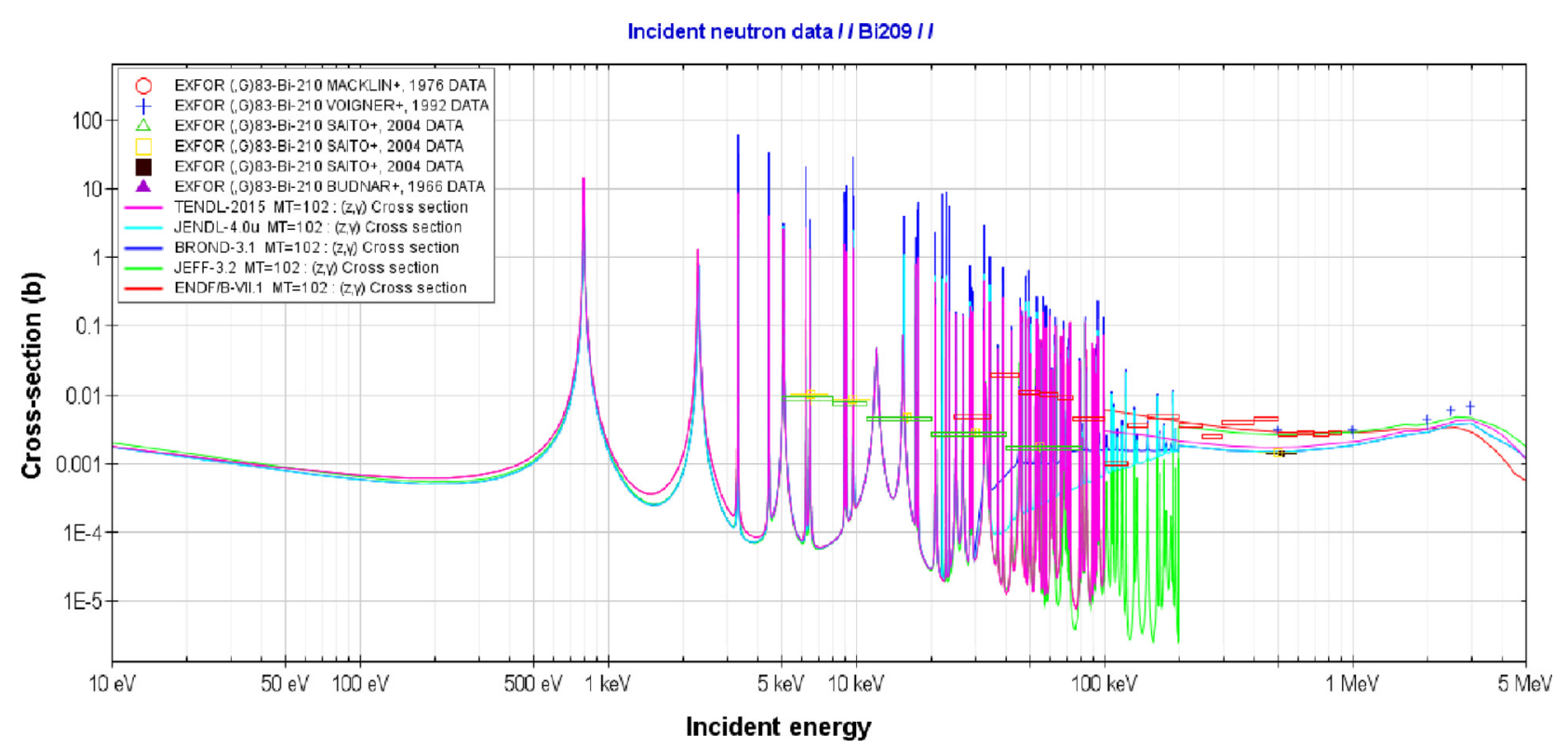

Fig. 2. ${ }^{209} \mathrm{Bi}$ (n, $\gamma$ ) cross section data: absolute values.

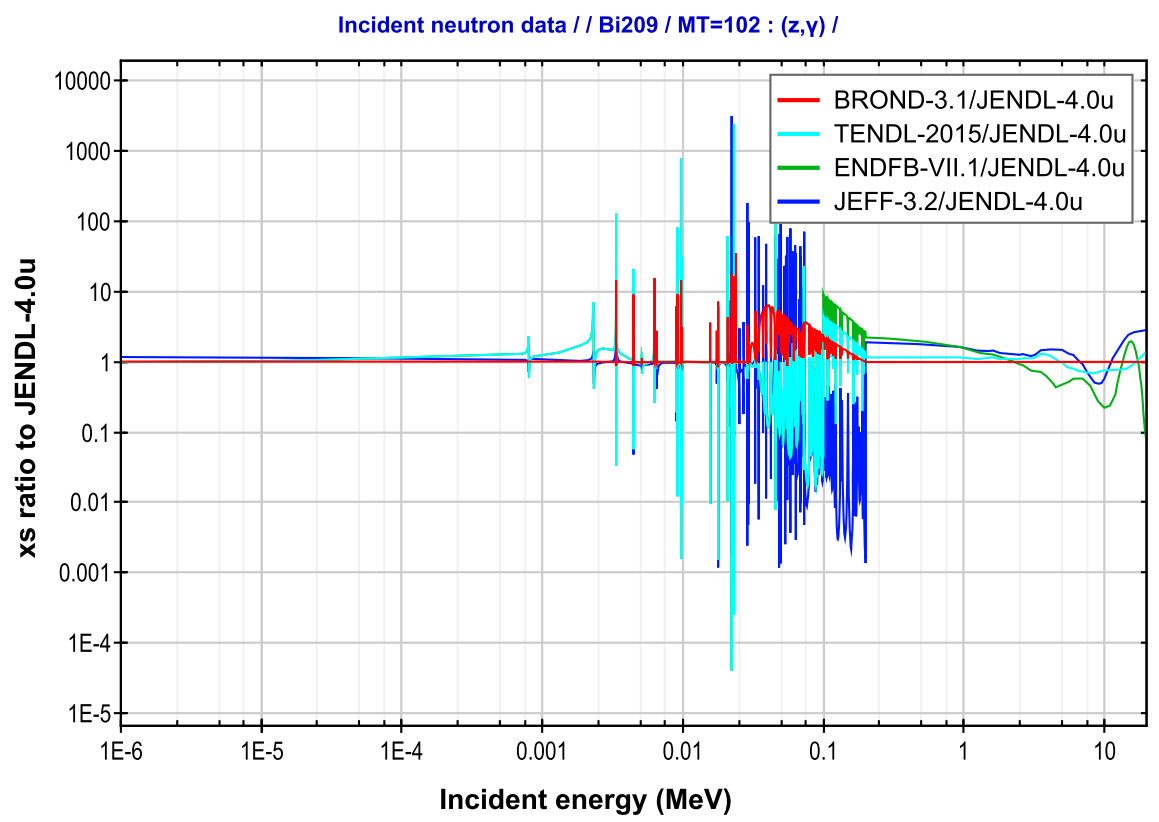

Fig. 3. ${ }^{209} \mathrm{Bi}(\mathrm{n}, \gamma)$ cross section data: ratios to JENDL-4.0.

and differences in the evaluations. Figure 2 shows the cross sections these libraries. Also, a ratio of each cross section to the JENDL-4.0 evaluation is shown in Figure 3 to enhance the contrast.

\subsection{JEFF-3.2}

The JEFF-3.2 file inherits most of its data from the JEFF-3.1 evaluation. JEFF-3.1 adopts the resolved resonance parameters below $200 \mathrm{keV}$ from JENDL-3.3, based on [15]. Modifications of JEFF-3.2 from JEFF-3.1 include an update of the resonance parameters using Mutti values [16] (based on the capture measurements at GELINA) and the values from the Atlas of neutron resonances [17] for parameters not provided by Mutti. No unresolved resonance formalism was used. The total cross section below $6.5 \mathrm{MeV}$ was adopted from the ENDF/B-VI.8 library. All other data were produced with the TALYS code [18] with model parameters adjusted to reproduce the existing experimental data. The background contribution to the elastic cross section in the resolved resonance region (RRR) present in JEFF-3.1 was set to zero.

\subsection{ENDF/B-VII.1}

The ENDF/B-VII.1 file adopts the cross section data and resolved resonance parameters from the ENDF/B-VI evaluation. The resolved resonance region extends up to 
$100 \mathrm{keV}$. Its resonance parameters were taken from Mughabghab [15] with modified scattering radius to fit experimental data. Above $100 \mathrm{keV}$, the energy dependent cross section is given, evaluated on numerous experimental data. Despite significant fluctuations in the cross section the unresolved resonance formalism was not used, which might lead to biased results close to $100 \mathrm{keV}$ in combination with strong self-shielding. A background contribution to the cross section above $99.46 \mathrm{keV}$ is present in the RRR.

\subsection{JENDL-4.0}

The JENDL-4.0 evaluation was adopted from JENDL-3.0 with some modifications. The resonance parameters (RRR below $200 \mathrm{keV}$ ) from Mughabghab [15] were updated taking into account the Domingo-Pardo et al. data [19] measured at $\mathrm{n}$ _TOF. A bound state was introduced to reproduce the thermal capture cross section from Mughabghab [17]. In the RRR a background cross section was added. Above the RRR, no unresolved resonance formalism was used and the capture cross section was normalized at $500 \mathrm{keV}$ to the measurements by Saito et al. [20] based on TOF measurements at the Pelletron accelerator in Tokyo.

\subsection{TENDL-2015}

The parameters for the resolved resonance region (limited to $100 \mathrm{keV}$ ) were taken directly from ENDF/B-VII.1. No unresolved resonance formalism was used. The TENDL2015 cross sections were re-evaluated using the TALYS [18] software and possibly renormalized to experimental data. Between 100 and $200 \mathrm{keV}$, the energy dependence of the total cross section seems nonphysical.

\subsection{BROND-3.1}

BROND-3.1 adopted the JENDL-3.3 data for the resolved resonance region, with the exception of the bound state that was not included. Hence, the thermal capture cross section was slightly modified compared to the Mughabghab [17] evaluation. A smooth background was added to the capture cross section in the resolved resonance range between 30 and $200 \mathrm{keV}$ to compensate the loss of the resonances. The continuous-energy cross section was also taken from JENDL.

\section{Current status of the branching ratio of the ${ }^{209} \mathrm{Bi}(\eta, \gamma){ }^{210 \mathrm{~g}} \mathrm{Bi} /{ }^{210 \mathrm{~m}} \mathrm{Bi}$}

To correctly predict the production of ${ }^{210} \mathrm{Po}$ the accurate knowledge of the branching ratio for the reactions ${ }^{209} \mathrm{Bi}$ $(\eta, \gamma){ }^{210 \mathrm{~g}} \mathrm{Bi} /{ }^{210 \mathrm{~m}} \mathrm{Bi}$ is essential. Unfortunately, branching ratios are difficult to be accurately measured by the TOF method. Therefore, the availability of accurate and reliable data is very limited. A comparison of some evaluations with experimental measurements is shown in Figure 4.

\subsection{JEFF-3.2}

The JEFF-3.2 branching ratios were updated from the previous JEFF-3.1 evaluation. The branching ratio does not show any energy dependence up to $1 \mathrm{MeV}$, with a

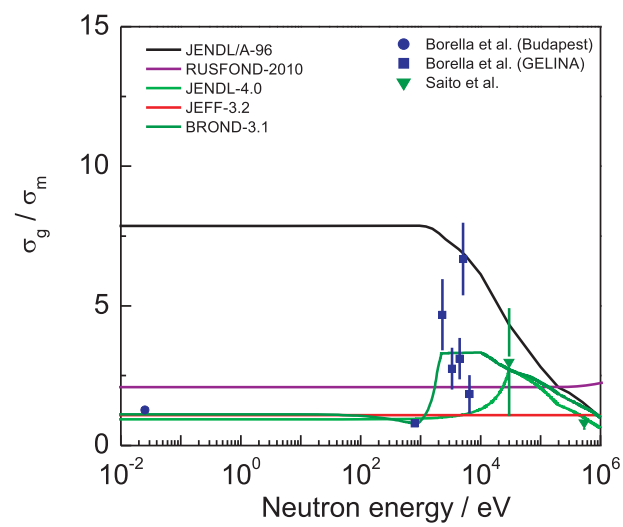

Fig. 4. ${ }^{209} \mathrm{Bi}$ ratio $\sigma_{\mathrm{g}} / \sigma_{\mathrm{m}}$.

constant value that is only few percents smaller than the Borella et al. [21] experimental values (feeding $\gamma$ rays) at thermal energy. Above $1 \mathrm{MeV}$ the existing trend is reversed, with the branching ratio to the meta state mostly being slightly more enhanced.

\subsection{ENDF/B-VII.1}

ENDF/B-VII.1, as well as the previous ENDF/B releases, does not provide any information on the branching ratios. Such a lack of data might lead to a critical overestimation of the ${ }^{210}$ Po production by those evolution codes that interpret the missing data as the absence of the ${ }^{210} \mathrm{Bi}$ meta state.

\subsection{JENDL-4.0}

JENDL-4.0 introduced the isomeric ratio in the thermal region from the table of Mughabghab [17]. The calculated data at $30 \mathrm{keV}$ are consistent with the experimental measurement by Saito et al. [22].

\subsection{BROND-3.1}

BROND-3.1 reproduces the strong energy-dependence of the isomeric ratio in all energy ranges by taking into account the latest experimental data including both Borella et al. [23] results for the $801.6 \mathrm{eV}$ resonance and the Saito et al. [20] measurements.

\section{Parametric study of the library performances}

A parametric study was carried out to assess whether the ${ }^{210}$ Po content prediction after a MYRRHA irradiation cycle in sub-critical mode was affected by the choice of the nuclear data library used for the calculation. The irradiation cycle of 90 days, with a constant core power of $70 \mathrm{MW}_{\text {th }}$, was simulated with the ALEPH-2 burnup code [24] developed at SCK•CEN. Capture cross sections first and branching ratios later were sequentially replaced from different evaluations into the base library ENDF/B-VII.1. The analysis of the results is reported in Table 2.

The largest difference in the ${ }^{210} \mathrm{Po}$ concentration obtained by replacing nuclear data from different libraries is $46 \%$ for cross sections and $63 \%$ for branching 
Table 2. Relative difference between the ${ }^{210} \mathrm{Po}$ concentrations. The value obtained with each library by replacing either $(\eta, \gamma)$ cross sections or branching ratios is compared to the ${ }^{210} \mathrm{Po}$ concentration obtained when averaging the results of all the libraries. MAX-MIN is the largest difference in ${ }^{210} \mathrm{Po}$ obtained with two libraries.

\begin{tabular}{lcr}
\hline Eval. & $\begin{array}{c}\text { Cross section } \\
\text { effect }(\%)\end{array}$ & $\begin{array}{c}\text { Branching ratio } \\
\text { effect }(\%)\end{array}$ \\
\hline JEFF-3.2 & -12 & -25 \\
ENDF/B-VII.1 & 21 & 45 \\
JENDL-4.0 & -19 & -16 \\
TENDL-2015 & 27 & 7 \\
BROND-3.1 & -16 & -12 \\
MAX-MIN & 46 & 70 \\
STDEV & 22 & 28 \\
\hline
\end{tabular}

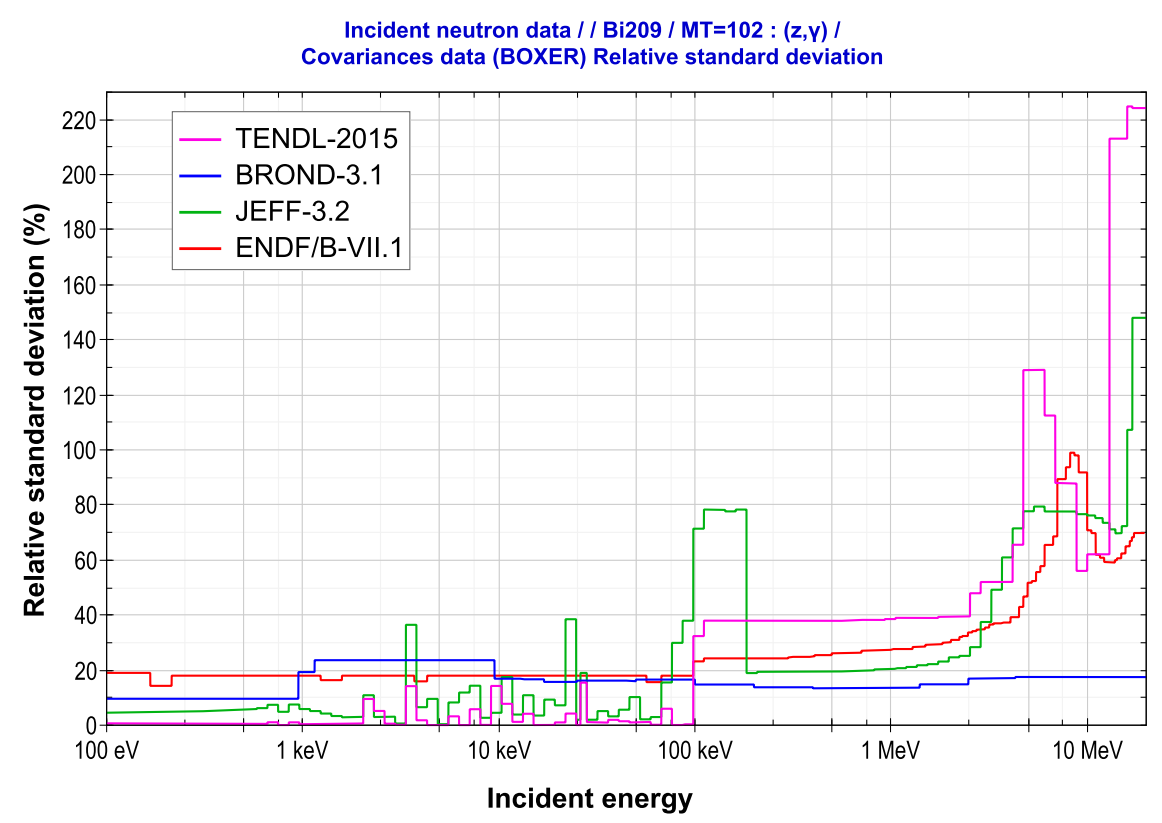

Fig. 5. Relative uncertainty of the ${ }^{209} \mathrm{Bi}$ cross section from different nuclear data libraries as a function of incident neutron energy.

ratios. Such deviations suggest inconsistencies between the existing data evaluations, which lead to major uncertainties when predicting the polonium content in MYRRHA.

\section{Nuclear data uncertainty propagation}

The covariance matrices found in the nuclear data libraries were propagated to quantify the uncertainty of the ${ }^{210} \mathrm{Po}$ content in the MYRRHA subcritical core. The uncertainty propagation was performed using ALEPH-2 in combination with the Monte Carlo sampling code SANDY [25]. SANDY was used to sample random cross sections from each ${ }^{209} \mathrm{Bi}$ nuclear data file. Together, the samples reproduced the information contained in the covariance sections of the corresponding file.

Figures 5 and 7 present the cross section covariances available in the nuclear data files used for this study. The plots were produced by the JANIS tool and display the correlation matrices collapsed on a 238-group energy grid by the ERRORR module of NJOY [26]. From the uncertainty profiles it is evident that BROND and ENDF/B provide a larger cross section uncertainty (about $20 \%$ ) compared to JEFF and TENDL up to about $100 \mathrm{keV}$. At $100 \mathrm{keV}$ and beyond the trend is inverted, with JEFF reaching a relative uncertainty peak of almost $80 \%$. Concerning the correlation matrices, JEFF, ENDF and TENDL show zero correlation between the resonance and fast regions as the two blocks were evaluated independently using different models. On the other hand, BROND includes long-range correlations based on the assimilation of systematic uncertainties.

For the JEFF and TENDL libraries, both resonance parameters and cross sections were sampled since both data types contained covariances (respectively sections MF32 and MF33 of a ENDF-6 file [27]). BROND and $\mathrm{ENDF} / \mathrm{B}$ only contained covariance matrices for the cross sections. It is relevant to mention that the ENDF/B covariance matrix for ${ }^{209} \mathrm{Bi}$ neutron capture cross section is far from being positive-definite, which means that not only it does not conform to the condition for Monte Carlo 


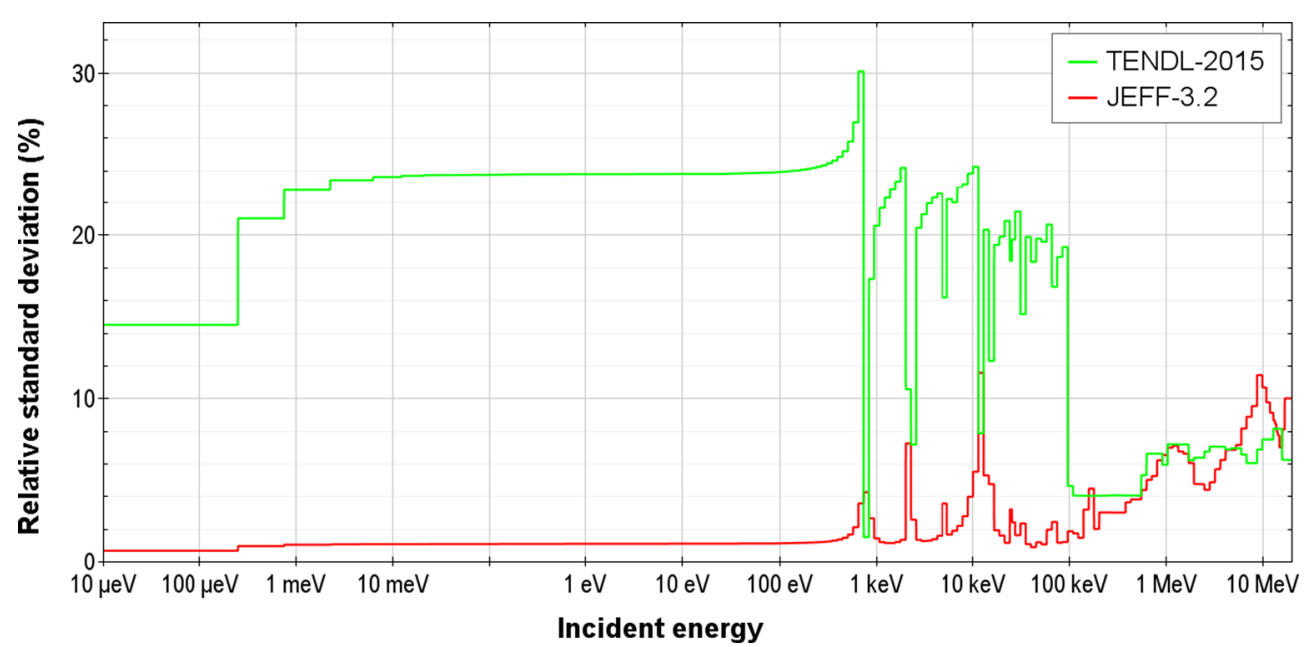

Fig. 6. Relative uncertainty of the ${ }^{209} \mathrm{Bi}(n, n)$ cross section from different nuclear data libraries as a function of incident neutron energy.

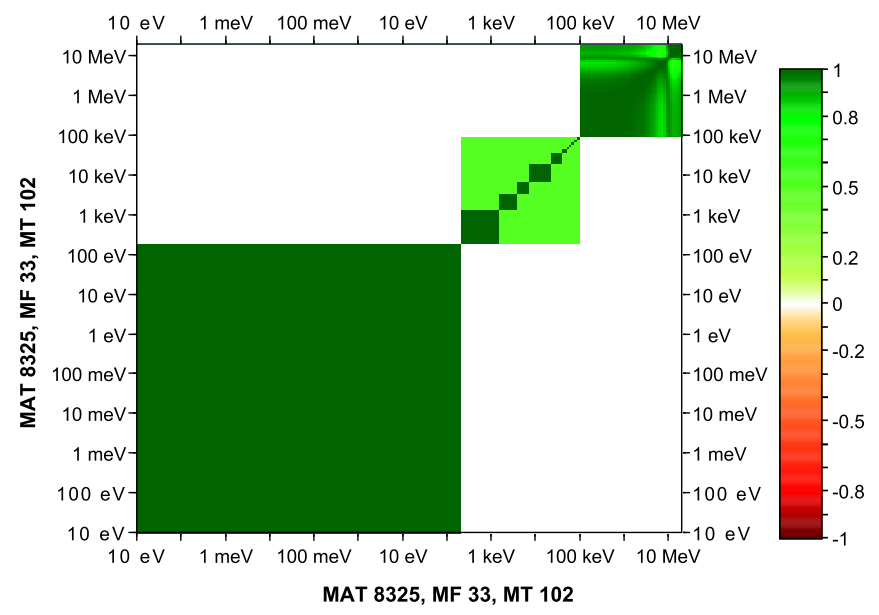

(a) ENDF/B-VII.1

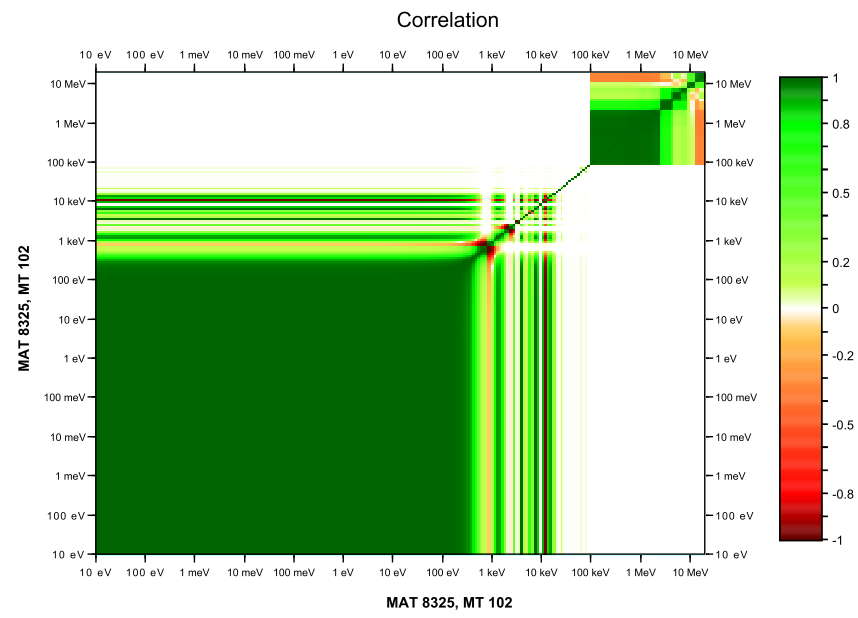

(c) TENDL-2015

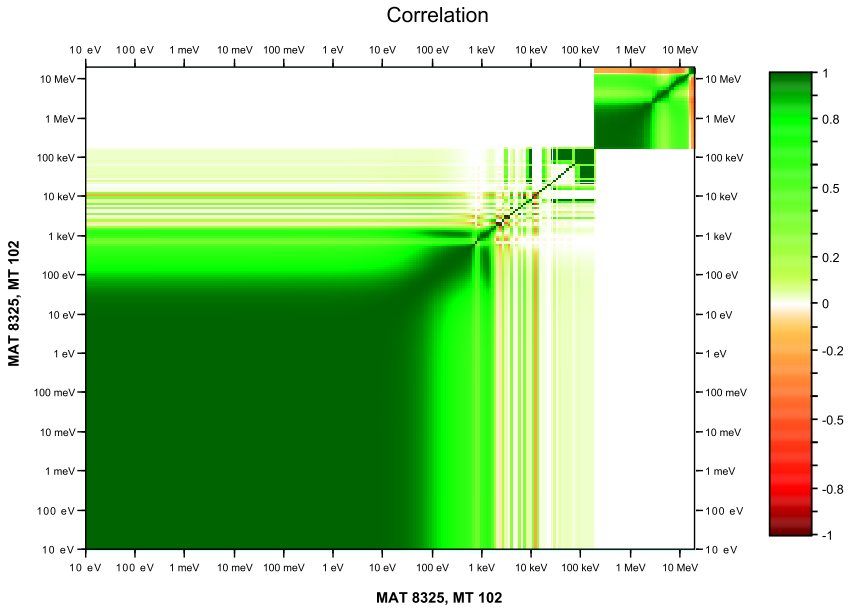

(b) JEFF-3.2

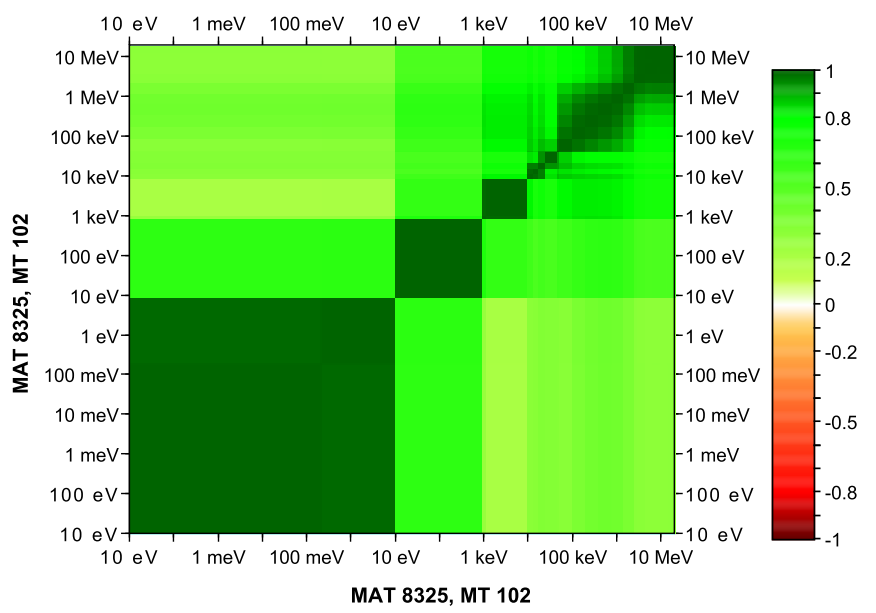

(d) BROND-3.1

Fig. 7. Correlation matrices of the ${ }^{209} \mathrm{Bi}(\mathrm{n}, \gamma)$ cross section. 
Table 3. Nuclear data uncertainty contribution to the ${ }^{210}$ Po production and the integrated flux.

\begin{tabular}{lccc}
\hline Eval. & Data & ${ }^{210} \mathrm{Po}(\%)$ & Flux $(\%)$ \\
\hline JEFF-3.2 & xs & 5.2 & 1.4 \\
JEFF-3.2 & res + xs & 5.9 & 2.1 \\
ENDF/B-VII.1 & xs & 10.9 & 2.6 \\
TENDL-2015 & res + xs & 21.6 & 29.7 \\
BROND-3.1 & xs & 11.8 & 1.7 \\
\hline
\end{tabular}

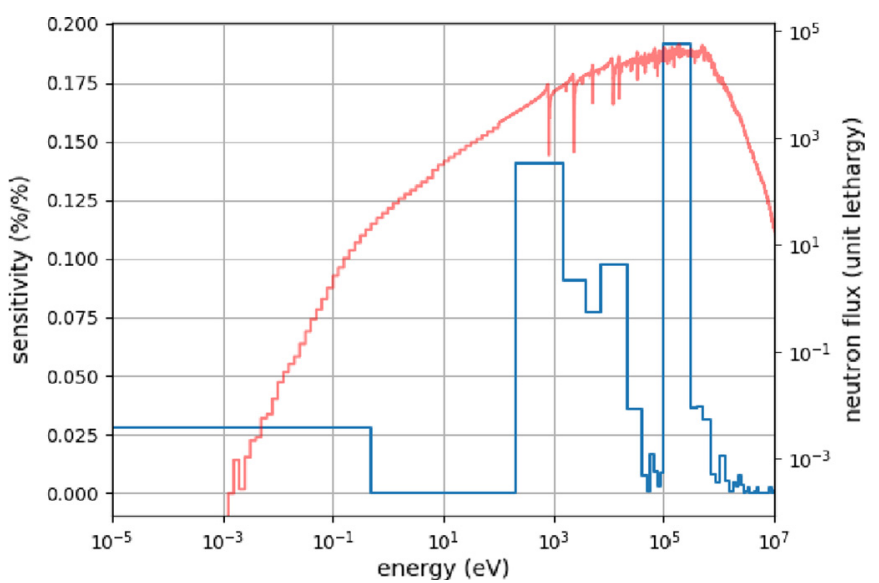

Fig. 8. ${ }^{210} \mathrm{Po}$ production sensitivity to the ${ }^{209} \mathrm{Bi}$ capture cross section and neutron flux as a function of incident neutron energy.

sampling but it is also mathematically inconsistent. The bias introduced in the samples to work around this issue could impact significantly on the results.

The calculated uncertainties on the ${ }^{210} \mathrm{Po}$ concentrations are summarized in Table 3 together with the corresponding calculated uncertainty on the integrated neutron flux. The influence of the capture cross section covariances on the polonium production ranges between $5.2 \%$ for JEFF and $21.6 \%$ from TENDL. Since only the $(\eta, \gamma)$ cross section data were perturbed for BROND and ENDF/B and because of the magnitude of such a reaction in the region of intense flux, the effect on the integrated flux is small. The energy self-shielding strongly anticorrelates (up to $-90 \%$ ) the integrated flux and the reaction rate of ${ }^{210} \mathrm{Bi}$, which is proportional to the ${ }^{210} \mathrm{Po}$ production. For TENDL and JEFF, the resonance parameters are also perturbed including correlations between capture and neutron widths. Consequently, the large uncertainty given in TENDL (Fig. 6) for the elastic scattering cross section, which is the dominant reaction with ${ }^{209} \mathrm{Bi}$, has a direct bearing on the energy-shift of the neutron flux and generates considerable uncertainties both on the integrated flux and on the ${ }^{210} \mathrm{Po}$ production. Contrary to the previous case, the two responses are now almost fully correlated.

\section{Sensitivity analysis via linear regression}

A global approach to the sensitivity analysis of ${ }^{210} \mathrm{Po}$ was performed based on Monte Carlo sampling and linear regression. Despite being less efficient in computer time, such an approach does not require programming adjoint function constructs into the code, as it would be done for a derivative-based approach. ALEPH-2, not having derivative-based sensitivity capabilities, can only take advantage from this global approach, since its application is straightforward.

Let us take a set of sampled input data in the form of perturbation coefficients $\mathbf{P}$, such as the perturbations generated by SANDY for the cross sections:

$$
P=\left[\begin{array}{ccc}
p_{1}^{(1)} & \cdots & p_{m}^{(1)} \\
\vdots & \ddots & \vdots \\
p_{1}^{(n)} & \cdots & p_{m}^{(n)}
\end{array}\right]
$$

where $m$ is the number of groups found in the cross section covariance matrix and $n$ is the number of samples. $p_{i \bullet}=p_{1}^{(i)}, \ldots, p_{m}^{(i)} \quad$ is the $i$ th line of the above matrix. These perturbation coefficients are used by SANDY to generate a perturbed cross section file that, when included in the ALEPH-2 calculation, it generates a response $r^{(\mathrm{i})}$. After running ALEPH-2 for the $n$ sample cases, a vector $\mathbf{R}$ of responses is produced:

$$
\mathbf{R}=\left[\begin{array}{c}
r^{(1)} \\
\vdots \\
r^{(n)}
\end{array}\right]
$$

As a part of the global sensitivity approach used in this work, we introduced a linear regression model of the type

$$
\mathbf{R}=\mathbf{P S}+\boldsymbol{\varepsilon}
$$

One thousand samples were used to determine the sensitivity coefficients $\mathbf{S}=\left[s_{1}, \ldots, s_{m}\right]^{T}$ of the response with respect to the cross sections in $m$ energy groups, by minimizing the residues $\boldsymbol{\varepsilon}$ with an ordinary least-square technique, as

$$
\mathbf{S}=\left(\mathbf{P}^{T} \mathbf{P}\right)^{-1} \mathbf{P}^{T} \mathbf{R} .
$$

The sensitivity profile is presented in Figure 8, overlaid on the spatial-averaged MYRRHA flux. The ${ }^{210} \mathrm{Po}$ production in MYRRHA exhibits a significant sensitivity to the ${ }^{209} \mathrm{Bi}$ capture cross section in correspondence of the peak of the flux (at about $100 \mathrm{keV}$ ) and of the first ${ }^{209} \mathrm{Bi}$ resonances. 


\section{Conclusion}

An analysis of the ${ }^{209} \mathrm{Bi}$ evaluated files from the JEFF-3.2, ENDF/B-VII.1, BROND-3.1, TENDL-2015 and JENDL4.0 libraries was carried out. In particular, the investigation covered those data types that are involved in the polonium production from ${ }^{209} \mathrm{Bi}$ activation. Significant differences were observed in the cross sections and branching ratios, which were proved to pose a severe constraint for the correct ${ }^{210} \mathrm{Po}$ content prediction in reactors that use the LBE technology, such as MYRRHA.

The covariance matrices found in the ${ }^{209} \mathrm{Bi}$ evaluations considered in this study exhibit different uncertainty profiles and energy correlations. The resulting uncertainty (intended as one standard deviation) on the ${ }^{210} \mathrm{Po}$ content after one irradiation cycle was quantified between $5 \%$ and $20 \%$, depending on which library was used. This level of uncertainty does not cover the deviation between ${ }^{210}$ Ponominal concentration values assessed with the evaluated files coming from different libraries, even when three standard deviations are considered. Furthermore, branching ratio covariances were not propagated since they are not provided in the evaluated files, although they are likely to add a significant uncertainty contribution to the polonium content.

The results obtained in this work call attention to the ${ }^{209} \mathrm{Bi}$ evaluations and uncertainties. Significant efforts are necessary to reduce the discrepancies between evaluated data. In particular, accurate measurements should be carried out to increase our knowledge of the ${ }^{209} \mathrm{Bi}$ activation cross section and the branching ratio, not only at thermal energy and for the first resonance at about $800 \mathrm{eV}$, but also in the energy ranges of interest for LBE reactors such MYRRHA, as made clear by the sensitivity plots. Because of the disagreement between libraries, a consistent evaluation for the branching ratios should be elaborated that also includes the energy-dependent behavior in the resonance region and above. This evaluation should be supported by additional experimental measurements, since the existing databases only contain a few data point.

\section{References}

1. H. Ait Abderrahim, P. Baeten, D. De Bruyn, R. Fernandez, MYRRHA - a multi-purpose fast spectrum research reactor, Energy Convers. Manage. 63, 4 (2012)

2. G. Van den Eynde, E. Malambu, A. Stankovskiy, R. Fernandez, P. Baeten, An updated core design for the multi-purpose irradiation facility MYRRHA, J. Nucl. Sci. Technol. 52, 1053 (2015)

3. OECD/NEA, Handbook on Lead-bismuth Eutectic Alloy and Lead Properties, Materials Compatibility, Thermalhydraulics and Technologies, Technical Report NEA. No. 7268, OECD/NEA, 2015

4. ICRP, Dose Coefficients for Intakes of Radionuclides by Workers, ICRP Publication 68, Ann. ICRP 24(4), 1994

5. ICRP, Age-dependent Doses to the Members of the Public from Intake of Radionuclides - Part 5 Compilation of Ingestion and Inhalation Coefficients, ICRP Publication 72, Ann. ICRP 26(1), 1995
6. S. Heinitz, J. Neuhausen, D. Schumann, Alkaline extraction of polonium from liquid lead bismuth eutectic, J. Nucl. Mater. 414, 221 (2011)

7. A. Stankovskiy, Y. Celik, G. Van den Eynde, A. Marchix, Identification of critical parameters related to the radioactive nuclide production and shielding of beam line, beam dump and spallation target module, Technical Report FP7 CHANDA Deliverable D11.1, SCK•CEN/5900250, $\mathrm{SCK} \bullet \mathrm{CEN}, 2015$

8. A. Stankovskiy, L. Fiorito, Residual heating calculations for MYRRHA, Technical Report SCK•CEN/4149434, SCK•CEN, 2015

9. L. Ortiz Amaya, J. Braet, Purification of Lead-Bismuth Eutectic used in accelerator driven systems, in Proceedings of the International Conference WM2009 (2009)

10. A.J. Koning, E. Bauge, J. Dean, E. Dupont, U. Fischer, R.A. Forrest, R. Jacqmin, H. Leeb, M.A. Kellett, R.W. Mills, C. Nordborg, M. Pescarini, Y. Rugama, P. Rullhusen, Status of the JEFF nuclear data library, in Proceedings of the International Conference on Nuclear Data for Science and Technology Jeju Island, Korea (2010), p. 1057

11. M.B. Chadwick, M. Herman, P. Oblozinsky, M.E. Dunn, Y. Danon, A.C. Kahler, D.L. Smith, B. Pritychenko, G. Arbanas, R. Arcilla, R. Brewer, D.A. Brown, R. Capote, A.D. Carlson, Y. S. Cho, H. Derrien, K. Guber, G.M. Hale, S. Hoblit, S. Holloway, T.D. Johnson, T. Kawano, B.C. Kiedrowski, H. Kim, S. Kunieda, N.M. Larson, L. Leal, J.P. Lestone, R.C. Little, E.A. McCutchan, R.E. MacFarlane, M. MacInnes, C.M. Mattoon, R.D. McKnight, S.F. Mughabghab, G.P.A. Nobre, G. Palmiotti, A. Palumbo, M.T. Pigni, V.G. Pronyaev, R.O. Sayer, A.A. Sonzogni, N.C. Summers, P. Talou, I.J. Thompson, A. Trkov, R.L. Vogt, S.C. van der Marck, A. Wallner, M.C. White, D. Wiarda, P.G. Young, ENDF/B-VII.1 nuclear data for science and technology: cross sections, covariances, fission product yields and decay data, Nucl. Data Sheets 112, 2887 (2011)

12. K. Shibata, O. Iwamoto, T. Nakagawa, N. Iwamoto, A. Ichihara, S. Kunieda, S. Chiba, K. Furutaka, N. Otuka, T. Ohsawa, T. Murata, H. Matsunobu, A. Zukeran, S. Kamada, J. Katakura, JENDL-4.0: a new library for nuclear science and engineering, J. Nucl. Sci. Technol. 48, 1 (2011)

13. A.J. Koning, D. Rochman, Modern nuclear data evaluation with the TALYS code system, Nucl. Data Sheets 113, 2841 (2012)

14. A.I. Blokhin, E.V. Gai, A.V. Ignatyuk, I.I. Koba, V.N. Manokhin, V.N. Pronyaev, New version of neutron evaluated data library BROND-3.1, Technical Report Yad.Reak. Konst.No.2, p.62, A.I. Leypunsky Institute for Physics and Power Engineering, Obninsk, Russia, 2016

15. S.F. Mughabghab, M. Divadeenam, N.E. Holden, Neutron resonance parameters and thermal cross sections, Number v. 1, pt. 1, Neutron Cross Sections Series (Academic Press, 1981)

16. P. Mutti, s-Process implications of ${ }^{136} \mathrm{Ba},{ }^{208} \mathrm{~Pb}$ and ${ }^{209} \mathrm{Bi}$ stellar capture rates, Technical report, 1997

17. S. Mughabghab, Atlas of neutron resonances: resonance parameters and thermal cross sections $Z=1-100$ (Elsevier, 2006)

18. A.J. Koning, D. Rochman, Modern nuclear data evaluation with the talys code system, Nucl. Data Sheets 113, 2841 (2012)

19. C. Domingo-Pardo, U. Abbondanno, G. Aerts, H. Alvarez Pol, F. Alvarez-Velarde, S. Andriamonje, J. Andrzejewski, P. Assimakopoulos, L. Audouin, G. Badurek, P. Baumann, 
F. Becvar, E. Berthoumieux, F. Calvino, D. Cano-Ott, R. Capote, A. Carrillo de Albornoz, P. Cennini, V. Chepel, E. Chiaveri, N. Colonna, G. Cortes, A. Couture, J. Cox, M. Dahlfors, S. David, I. Dillman, R. Dolfini, W. Dridi, I. Duran, C. Eleftheriadis, M. Embid-Segura, L. Ferrant, A. Ferrari, R. Ferreira-Marques, L. Fitzpatrick, H. Frais-Koelbl, K. Fujii, W. Furman, R. Gallino, I. Goncalves, E. Gonzalez-Romero, A. Goverdovski, F. Gramegna, E. Griesmayer, C. Guerrero, F. Gunsing, B. Haas, R. Haight, M. Heil, A. HerreraMartinez, M. Igashira, S. Isaev, E. Jericha, Y. Kadi, F. Kappeler, D. Karamanis, D. Karadimos, M. Kerveno, V. Ketlerov, P. Koehler, V. Konovalov, E. Kossionides, M. Krticka, C. Lamboudis, H. Leeb, A. Lindote, I. Lopes, M. Lozano, S. Lukic, J. Marganiec, L. Marques, S. Marrone, P. Mastinu, A. Meng, New measurement of neutron capture resonances in ${ }^{209} \mathrm{Bi}$, Phys. Rev. C 74, 025807 (2006)

20. K. Saito, M. Igashira, J. Kawakami, T. Ohsaki, T. Obara, H. Sekimoto, Measurement of keV-neutron capture cross sections and capture gamma-ray spectra of ${ }^{209} \mathrm{Bi}$, J. Nucl. Sci. Technol. 41, 406 (2004)

21. A. Borella, T. Belgya, S. Kopecky, F. Gunsing, M. Moxon, M. Rejmund, P. Schillebeeckx, L. Szentmiklosi, Determination of the ${ }^{209} \mathrm{Bi}\left(\mathrm{n}\right.$, gamma) ${ }^{210} \mathrm{Bi}$ and ${ }^{209} \mathrm{Bi}(\mathrm{n}$, gamma $){ }^{210} \mathrm{~m}, \mathrm{~g} \mathrm{Bi}$ reaction cross sections in a cold neutron beam, Nucl. Phys. A 850, 1 (2011)
22. K. Saito, M. Igashira, T. Ohsaki, T. Obara, H. Sekimoto, Measurement of cross sections of the 210-Po production reaction by $\mathrm{keV}$-neutron capture of $209-\mathrm{Bi}$, in JAERI Conference proceeding (2003), Vol. 6, p. 133

23. A. Borella, T. Belgya, E. Berthoumieux, N. Colonna, C. Domingo-Pardo, J.C. Drohe, F. Gunsing, S. Marrone, T. Martinez, C. Massimi, P. Mastinu, P.M. Milazzo, P. Schillebeeckx, G. Tagliente, J. Tain, R. Terlizzi, R. Wynants, Measurements of the branching ratio of the ${ }^{209} \mathrm{Bi}(\mathrm{n}$, gamma) ${ }^{210 \mathrm{~g}} \mathrm{Bi} /{ }^{210 \mathrm{~m}} \mathrm{Bi}$ reactions at GELINA, in ND 2007 (EDP Sciences, 2007), No. 150

24. A. Stankovskiy, G. Van den Eynde, Advanced method for calculations of core burn-up, activation of structural materials, and spallation products accumulation in accelerator-driven systems, Sci. Technol. Nucl. Ins. 2012, 545103 (2012)

25. L. Fiorito, G. Zerovnik, A. Stankovskiy, G. Van den Eynde, P.E. Labeau, Nuclear data uncertainty propagation to integral responses using SANDY, Ann. Nucl. Energy 101, 359 (2017)

26. R.E. MacFarlane, D.W. Muir, R.M. Boicourt, A.C. Kahler, The NJOY nuclear data processing system, version 2012, Technical Report LA-UR-12-27079, LANL, 2012

27. CSEWG, ENDF-6 formats manual. Data formats and procedures for the evaluated nuclear data files ENDF/ B-VI and ENDF/B-VII (Upton, USA, 2013)

Cite this article as: Luca Fiorito, Alexey Stankovskiy, Augusto Hernandez-Solis, Gert Van den Eynde, Gasper Zerovnik, Nuclear data uncertainty analysis for the Po-210 production in MYRRHA, EPJ Nuclear Sci. Technol. 4, 48 (2018) 